\title{
Estudi de la Llei de Faraday-Lenz fent servir el mòbil i el sensor de voltatge
}

Víctor López Simó (victor.lopez@uab.cat) Centre de Recerca per a l'Educació Científica i Matemàtica (CRECIM) - Universitat Autònoma de Barcelona (UAB)

Presentem una experiència de laboratori de Física de Batxillerat enfocada a estudiar experimentalment la relació expressada en la llei de Faraday-Lenz. Llancem un imant per dins un tub de metacrilat que travessa una bobina, i comparem la FEM mesurada directament amb un sensor de voltatge i la FEM calculada a partir de les mesures obtingudes amb el mòbil: la posició de l'imant respecte la bobina en cada instant de temps $i$ el valor de la intensitat del camp magnètic per cadascuna d'aquestes posicions relatives. A partir de senzills càlculs numèrics, s'obtenen dues representacions gràfiques molt semblants, que poden ajudar a l'alumnat a fer la sempre complexa connexió entre electricitat i magnetisme.

Paraules clau: sensor voltatge, telèfon mòbil, imant, bobina, electromagnetisme.

We present an upper-secondary school physics laboratory experience focused on the study of the Faraday-Lenz law. We drop a piece of magnet through a methacrylate tube that crosses a coil, and we compare the measured EMF measured with the voltage sensor and the EMF calculated based on the mobile-based measurements: the magnet position over the time and the magnetic field strength for each of these positions. Using easy calculations, two similar representations can be obtained, helping students to build the difficult relationship between electricity and magnetism.

Keywords: Voltage sensor, mobile phone, magnet, coil, electromagnetism.

\section{INTRODUCCIÓ}

La Llei de Faraday-Lenz, o Llei d'Inducció Electromagnètica, és una de les relacions més rellevants i transcendents de la física clàssica, ja que expressa una relació entre electricitat i magnetisme. Aquesta relació sovint s'expressa amb la següent equació, on s'estableix la dependència entre un camp elèctric $E$ amb la variació d'un camp magnètic B:

$$
\oint_{c} E \cdot d l=-\frac{d}{d t} \int_{S} B \cdot d A
$$

Aquesta equació expressa que, donat el flux de camp magnètic que travessa una superfície $\mathbf{S}$ qualsevol, si aquest flux canvia al llarg del temps (ja sigui perquè canvia la intensitat del camp magnètic o bé perquè canvia pròpia superfície), es genera un camp elèctric al llarg del contorn $\mathbf{C}$ d'aquesta superfície, que es proporcional a la variació del flux magnètic.

Si bé expressat així pot semblar un tant abstracte, aquesta relació té una traducció molt concreta en el cas de les bobines: si es modifica d'alguna manera el camp magnètic que travessa una bobina (podem imaginar tota bobina com una superfície tancada, on el contorn d'aquesta superfície és el propi fil conductor enrotllat), la Força Electromotriu Induïda (FEM) que es genera entre els extrems de la bobina és proporcional a aquesta variació del camp magnètic. Això s'expressa com:

$$
\varepsilon=-N \frac{d \Phi_{B}}{d t}
$$


La inducció de FEM en bobines a partir de variacions de flux magnètic és la base del funcionament del tots els generadors electromagnètics, presents en la immensa majoria de processos de generació elèctrica del planeta. Si bé existeixen altres mecanismes per generar electricitat (les reaccions químiques d'oxidació reducció, els processos foto-elèctrics en semiconductors, etc.), el moviment relatiu entre un imant generador d'un camp B i una bobina per la que travessa un flux $\Phi_{\mathrm{B}}$ és la base per explicar com es genera electricitat tant a les grans centrals elèctriques (ja siguin de combustió, eòliques, hidràuliques, etc.) com en molts objectes quotidians (les dinamo de la bicicleta, les llanternes recarregables de manovella, etc.). Alhora, aquesta mateixa relació entre camp elèctric i variació de flux magnètic és també el principal fonament que explica el funcionament dels motors elèctrics, presents en una infinitat d'objectes quotidians (els electrodomèstics, les joguines, els transports, etc.).

\section{L'ENSENYAMENT I APRENENTATGE DE LA LLEI DE FARADAY-LENZ A SECUNDÀRIA}

La Llei de Faraday-Lenz simbolitza el vincle entre electricitat i magnetisme, dos camps fenomenològics que a secundària acostumen a presentar-se $\mathrm{i}$ estudiar-se per separat. Donada la rellevància que té aquest vincle, en els darrers anys s'han fet diferents propostes didàctiques per ajudar als estudiants a comprendre'l. Algunes propostes didàctiques per a la ESO posen la mirada en l'estudi i construcció de motors electromagnètics (Achón, 2017), en l'estudi qualitatiu de les aplicacions quotidianes de la inducció electromagnètica (Lorenzo, Codina, Sánchez, 2016) o en la mesura experimental de la FEM induïda en una bobina quan s'hi llença un imant, amb l'objectiu d'analitzar el paper que juguen variables com el nombre d'espires de la bobina, el tipus d'imant o la velocitat amb que travessa la bobina quan cau (López, Garrido Espeja i Pintó, 2016). Alhora, també existeixen propostes d'experiències pràctiques de Batxillerat per comprendre nocions d'electromagnetisme a partir d'imants i bobines (Folch i altres, 2014), així com propostes d'exercicis pràctics $i$ càlculs matemàtics (Jaén, 2009). Finalment, existeixen diferents propostes i recerques sobre aprenentatge de la inducció electromagnètica en els estudis universitaris de Física i Enginyeria (Guisasola, Almudi i Zuza, 2013), però que escapen de l'abast d'aquest article.
Dins d'aquest conjunt de propostes existents, trobem a faltar activitats que posin l'èmfasi en la relació experimental entre electricitat i magnetisme, que ajudin a l'alumnat a establir aquest vincle a partir de mesures que puguin ser fetes amb materials senzills de laboratori, i que ofereixin l'oportunitat de discutir la validesa de les equacions presentades anteriorment. Una manera de fer-ho és comparar la mesura experimental de la FEM que s'indueix en la bobina quan varia el flux magnètic que la travessa amb el càlcul de la derivada numèrica de la mesura experimental d'aquest flux magnètic.

A continuació presentem una experiència, pas a pas, de com es poden dur a terme aquestes mesures de la forma més senzilla $\mathrm{i}$ entenedora possible, fent servir material comú de laboratori instruments de mesura fàcilment disponibles a l'aula. L'enfocament didàctic amb que es presenti aquesta experiència a l'alumnat de Batxillerat pot ser variat, ja que pot incloure's en el treball per projectes, en un Treball de Recerca o en una pràctica de laboratori.

\section{DESCRIPCIÓ DE L’EXPERIÈNCIA}

\section{Muntatge experimental i instruments de mesura}

Proposem un senzill muntatge, compost per una bobina, un imant de neodimi i un tub de metacrilat pel que es deixarà caure l'imant (figura 1). La bobina $i$ el tub poden subjectar-se amb pinces, agafades a un peu de ferro de laboratori. Sobre la base del peu de ferro és convenient col-locar un material que esmorteeixi el cop de l'imant al caure.

Pel que fa als instruments de mesura, farem servir un sensor de voltatge connectat als extrems de la bobina i un telèfon mòbil per enregistrar en vídeo la caiguda de l'imant.

\section{Primera part: Mesura directa de la FEM induïda en la bobina}

El sensor de voltatge connectat als extrems de la bobina permet mesurar a temps real la variació de diferència de potencial entre els extrems de la bobina, és a dir, la FEM induïda. Al llençar l'imant, s'obté una gràfica com la de la figura 2. En aquesta gràfica s'observa un primer pic amb valors de FEM negatius (del segon 0,04 al 0,07), que correspon a la fracció de temps en que l'imant s'està acostant a la bobina i comença a travessar-la. El valor mínim de $-0,8 \mathrm{~V}$ correspon al moment on l'imant està començant a entrar a la bobina. De la mateixa 
manera, el pic positiu (del segon 0.07 al 0,10 ) correspon a la fracció de temps on l'imant està sortint de la bobina per sota, trobant el valor màxim de $0,8 \mathrm{~V}$ en el moment en que l'imant està sortint de la bobina.

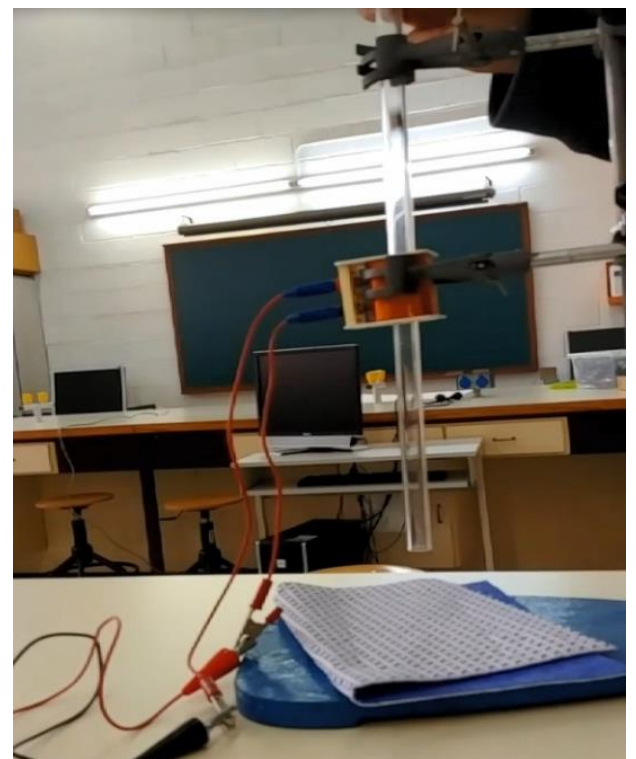

Figura 1. Muntatge experimental compost per una bobina, un imant i un tub de metacrilat, subjectes amb pinces de laboratori a un peu de ferro. La bobina està connectada a un sensor de voltatge.

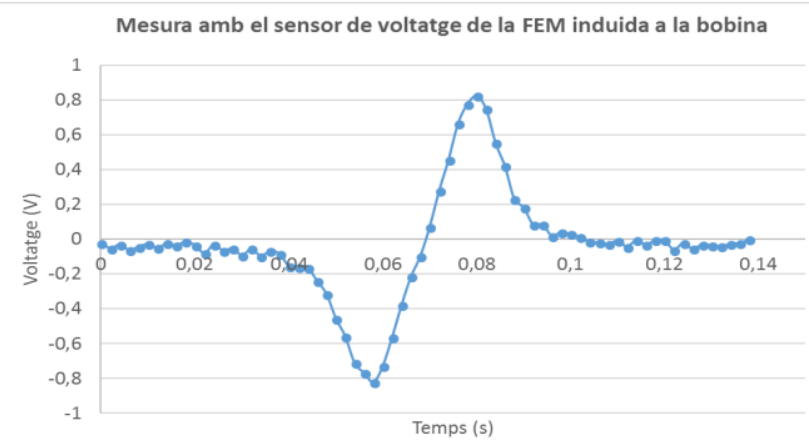

Figura 2. Gràfica obtinguda amb el sensor de voltatge de la FEM induïda en funció del temps.

\section{Segona part: Mesura indirecta de la intensitat de camp magnètic a l'interior de la bobina}

En paral-lel a aquesta mesura, amb el mòbil, s'enregistra en forma de vídeo el moviment de l'imant. Un cop enregistrat el vídeo, es pot fer servir un programa d'anàlisi de vídeo, com ara Tracker [1], per tal d'analitzar el moviment de l'imant. Aquest programa permet mesurar, fotograma a fotograma, el moviment i la trajectòria de qualsevol objecte mòbil. Per fer-ho, és extremadament important mantenir el mòbil totalment fixe (a poder ser, també amb pinces de laboratori), ja que qualsevol petit moviment del telèfon pot distorsionar la mesura d'aquesta posició. En el nostre cas, mesurem la distància entre el centre de l'imant i el centre de la bobina. Hem representat el valor d'aquesta distància en la figura 3 , on s'observa com en el període comprès entre 0,0 i 0,07 segons l'imant s'està acostant a la bobina amb un moviment que s'acosta al d'una caiguda lliure (MRUA), mentre que en la fracció de temps des de 0,07 fins a 0,14 segons l'imant s'allunya de la bobina amb un moviment que s'acosta al d'una caiguda amb velocitat terminal (MRU).

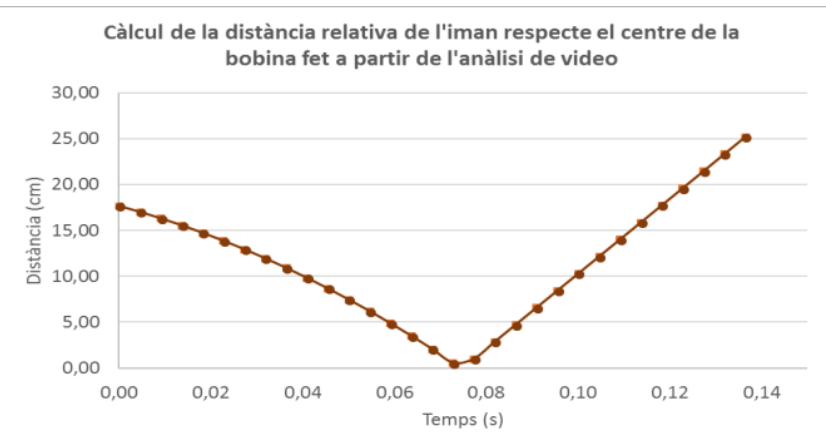

Figura 3. Gràfica obtinguda amb el programa Tracker després d'haver mesurat la distància relativa entre imant i bobina fotograma a fotograma.

La gràfica distància temps és la que permetrà mesurar, indirectament, quin ha estat el valor de la intensitat de camp magnètic a l'interior de la bobina en cada instant de temps. Per fer-ho, col-loquem el telèfon mòbil a una distància de l'imant que correspon a cada un dels valors de distància representats de la figura 3. Per fer-ho, es fa servir una cinta mètrica, tal com es presenta en la figura 4. La intensitat de camp magnètic (que es mesura en $\mu \mathrm{T}$ ) es pot mesurar amb una gran varietat d'apps gratuïtes per a l'ocasió (Torres, Bañon i López, 2017).

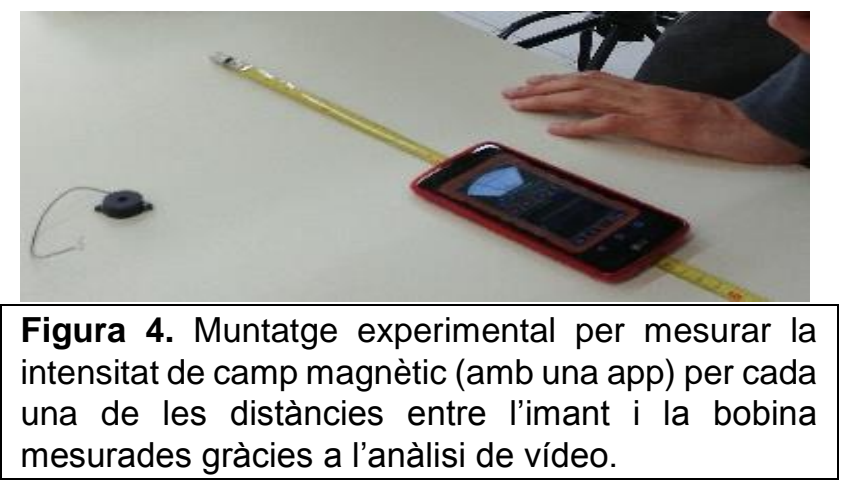


La mesura de la intensitat en funció de la distància, combinada amb la mesura de la distància en funció del temps, ens permet construir una gràfica de la intensitat de camp magnètic que hi hauria a l'interior de la bobina (degut a l'efecte de l'imant) en funció del temps. Al fer-ho, obtenim una gràfica com la de la figura 5 .

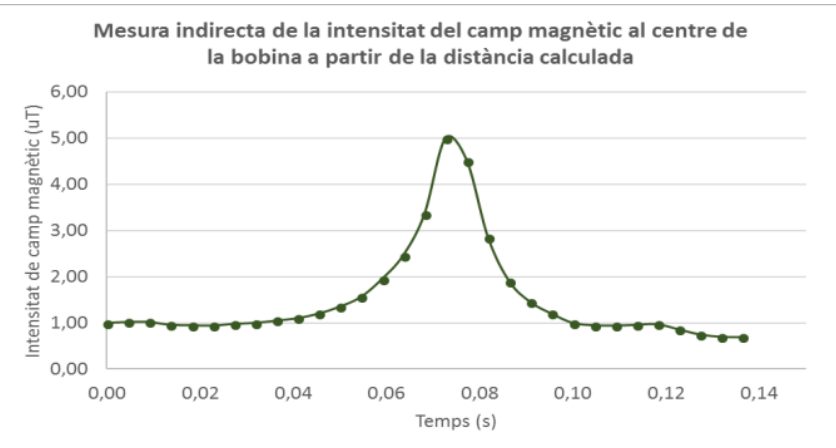

Figura 5. Gràfica obtinguda de forma indirecta, combinant la gràfica distància - temps amb al gràfica intensitat de camp - distància.

\section{Tercera part: Càlcul numèric de la variació del flux de camp magnètic en la bobina}

Arribats a aquest punt, només queda tornar a l'equació de la que partíem a l'inici, que expressava la relació:

$$
\varepsilon=-N \frac{d \Phi_{B}}{d t}
$$

La representació en funció del temps de la part esquerra de l'equació correspon a la gràfica de la figura 2, mentre que la representació en funció del temps de la part dreta de l'equació pot fer-se a partir dels valors exposats en la figura 5 . Fer fer-ho, no tindrem en compte ni el nombre d'espires $\mathrm{N}$ ni tampoc la superfície de la pròpia bobina, ja que es tracta de valors constants en el temps (no varien al llarg de la caiguda). Assumirem, per tant, que la funció $\Phi_{B}(t)$ és proporcional a la funció $B(t)$, i que per tant, el que calcularem és la funció derivada $B(t)$ en funció del temps. Aquest càlcul és fàcilment realitzable amb un full de càlcul, considerant que el valor de la funció $\mathrm{dB}(\mathrm{t}) / \mathrm{dt}$ és la diferència entre dos valors consecutius de $\mathrm{B}(\mathrm{t})$ dividits per dos valors consecutius de t (figura 6).

Al fer-ho, ens queda una gràfica com la de la figura 7, que té una clara semblança visual a la gràfica de la figura 2. Cal tenir en compte que la diferència entre el sentit positiu i negatiu dels pics calculats en la figura 7 respecte els mesurats a la figura 2 s'explica pel signe negatiu que relaciona les dues parts de l'equació.

\begin{tabular}{|l|l|l|l|}
\hline 4 & $\mathrm{~A}$ & $\mathrm{~B}$ & $\mathrm{C}$ \\
\hline 1 & $\mathrm{t}$ & $\mathrm{B}$ & $\mathrm{dB} / \mathrm{dt}$ \\
\hline 2 & 0,000 & 0,78 \\
\hline 3 & 0,005 & \\
\hline 4 & 0,010 & 0,81 & $=(\mathrm{B} 3-\mathrm{B} 2) /(\mathrm{A} 3-\mathrm{A} 2)$ \\
\hline 5 & 0,015 & 0,82 & 2 \\
\hline
\end{tabular}

Figura 6. Mètode numèric amb full de càlcul per obtenir la funció $\mathrm{dB}(\mathrm{t}) / \mathrm{dt}$ a partir de la funció $\mathrm{B}(\mathrm{t})$.

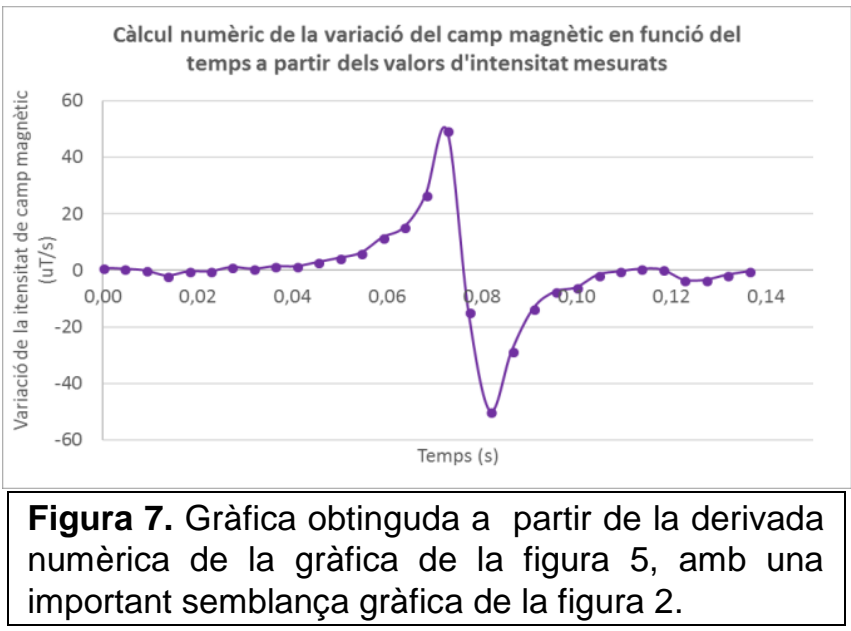

\section{LIMITACIONS DE L'EXPERIÈNCIA}

Malgrat l'interès didàctic que ofereix aquesta experiència degut a la simplicitat alhora que potència dels resultats que permet obtenir, també cal destacar algunes limitacions de l'experiència, que el professorat haurà de preveure abans de realitzar-la:

- És pràcticament impossible sincronitzar manualment el llançament de l'imant, la mesura de la FEM a través del sensor de voltatge i l'enregistrament del vídeo per mesurar la distància imant - bobina. Per tant, és convenient posar a enregistrar tant el sensor com el vídeo abans del llançament, i posteriorment ajustar els valors del temps amb el full de càlcul.

- Els moments en que l'imant està travessant la bobina, no se'l pot identificar en el vídeo, ja que està ocult. Per tant, és convenient que les dades obtingudes amb l'anàlisi de vídeo, es faci un ajust de les dades fent servir les equacions del 
moviment del MRUA i el MRU, que permeti interpolar els valors de la distància entre l'imant $i$ la bobina en aquest instant de temps.

- La caiguda de l'imant succeeix en un interval de temps molt curt (menys de 0,15 segons), i per tant, és convenient ajustar tant el sensor de voltatge com el vídeo amb el màxim de presa de dades per segon. En el cas del vídeo, si es disposa d'enregistrament "slow motion" pot incrementar la precisió de la mesura.

Finalment, també cal tenir en compte que el experiment requereix nombroses simplificacions de caràcter conceptual, que si bé són difícilment solucionables a nivell experimental, és important tenir presents. En primer lloc, l'experiment es basa en la mesura de la distància imant-bobina considerant el segment es fa des del centre de la bobina al centre de l'imant, cosa que és una simplificació important, ja que cada espira de la bobina està a una distància diferent de l'imant, no pas totes concentrades al centre. A més, hem negligit altres efectes electromagnètics que intervenen en el fenomen, com ara els corrents de Foucault o la pròpia resistència elèctrica de la bobina (que faria que el voltatge mesurat sigui lleugerament inferior a la FEM), etc.

\section{AGRAIIMENTS}

La motivació original per a dur a terme aquest experiment s'emmarca en les discussions fetes amb l'estudiant de Batxillerat Marc Seuba Pablo a través de la col-laboració del Programa Argó, entre la UAB i els centres educatius de secundària.

\section{BIBLIOGRAFIA}

ACHÓN , J. (2017) La moguda electromagnètica. Recurs didàctic publicat a ARC. http://apliense.xtec.cat/arc/node/30657

FOLCH, M., MONTANYÀ, G., NOTARIO, M., OLIVELLA, J., PONT, J., ROVIRA, M., VALLBÉ, M. I VILAJOSANA, M. D. (2014). Electricitat i magnetisme. Recursos de Física, 14.

GUISASOLA, J., ALMUDI, J.M., ZUZA, K. (2013). University Students' understanding of Electromagnetic Induction. International journal of Science Education, 35 (16), 2692-2717

LÓPEZ, V., GARRIDO ESPEJA, A.,PINTÓ, R. (2016). Generació d'electricitat per inducció electromagnètica. Seqüència didàctica per a l'estudi de la inducció electromagnètica. Barcelona: Publicacions CRECIM https://ddd.uab.cat/record/182193

LORENZO, J., CODINA, M. L., SÁNCHEZ, M. C. (2016). Electromangetisme. Recurs didàctic publicat a ARC. . http://apliense.xtec.cat/arc/node/30021

TORRES, A.L., BAÑÓN, D., LÓPEZ, V. (2017) Empleo de smartphones y apps en la enseñanza de la física y química. Enseñanza de las Ciencias, número Extraordiario 2017, pp: 671-677

[1] Tracker Video Analysis and Modeling Tool for Physics Education: https://physlets.org/tracker/ 